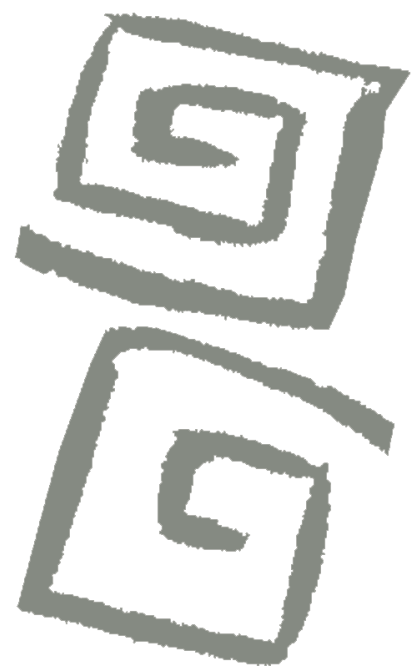

\title{
Análisis de errores con medicamentos en pacientes ingresados en salas de cirugía y recuperación posanestésica de un hospital de alta complejidad en Bogotá, Colombia
}

\author{
An analysis of medication errors in patients admitted \\ to surgery rooms and post-anesthetic recovery at a \\ high-complexity hospital in Bogota, Colombia
}

Ángel Humberto Villanueva', José Julián López Gutiérrez²

'Enfermero, Magíster en Farmacología. Docente, Universidad Surcolombiana, Bogotá, Colombia. $\triangle$ (iD)

${ }^{2}$ Autor de correspondencia. Farmacéutico, Doctor en Ciencias Farmacéuticas. Profesor Asociado, Universidad Nacional de Colombia, Bogotá, Colombia. $\bowtie$ (iD)
RESUMEN Los errores con medicamentos representan una de las principales causas de incidentes y eventos adversos durante el periodo perioperatorio por lo que en este estudio se analizaron los errores antes, durante y después de la administración de anestesia general para la realización de cirugía abdominal en un hospital de alta complejidad en Bogotá, Colombia. Se realizó un estudio descriptivo de corte transversal en 390 pacientes entre los meses de enero a septiembre de 2019. De las 3.677 administraciones de medicamentos, en el $60 \%$ se incurrió en algún tipo de error, mayoritariamente en cirugías de urgencia. El grupo farmacológico con más errores fue el de los anestésicos generales con un $32 \%$. Todos los errores identificados se configuraron como situaciones con potencial de daño lo que indica la necesidad de promover la estandarización de actividades durante la utilización de medicamentos y la cultura de seguridad asistencial para evitar que ocasionen eventos adversos.

PALABRAS CLAVES Errores de Medicación; Quirófanos; Seguridad del Paciente; Efectos Colaterales y Reacciones Adversas Relacionados con Medicamentos; Colombia.

\begin{abstract}
Medication errors represent one of the main causes of incidents and adverse events during the perioperative period. Therefore, this study analyzes errors before, during, and after the administration of general anesthesia for abdominal surgery at a high-complexity hospital in Bogota, Colombia. A descriptive cross-sectional study was conducted with 390 patients between January and September 2019. Of the 3,677 medication administrations, some type of error was made in $60 \%$ of cases, mostly in emergency surgeries. The pharmacological group with the most errors was general anesthetics, with $32 \%$. All identified errors constituted situations with harm potential, indicating the need to promote the standardization of activities involving the use of medications and a culture of healthcare safety in order to avoid adverse events.
\end{abstract}

KEY WORDS Medication Errors; Operating Room; Patient Safety; Drug-Related Side Effects and Adverse Reactions; Colombia. 


\section{INTRODUCCIÓN}

Las instituciones prestadoras de servicios de salud se enfocan en la integración de servicios médicos, farmacéuticos, cuidados de enfermería, administración y hotelería para solucionar con calidad y calidez los problemas de salud de un individuo o una población. Sin embargo, la creciente complejidad de la atención asistencial y la vulnerabilidad de los pacientes han favorecido la presencia de incidentes y eventos adversos que limitan el logro de los objetivos prevenir, curar y/o rehabilitar, promoviendo en los últimos años la incursión de la seguridad clínica como uno de los componentes esenciales de la calidad en salud ${ }^{(1,2)}$.

Desde la publicación de la primera edición, en 1860, de Notes on nursing de Florence Nightingale, pasando por el documento histórico To err is human: building a Safer Health System en 1999, hasta la actualidad, el interés por la seguridad clínica ha presenciado un aumento gradual, que ocupan cada vez mayor espacio en las políticas de gobiernos y hospitales, motivados por mejorar la calidad de la atención asistencial ${ }^{(3)}$.

Los incidentes y eventos adversos ocurridos durante la atención sanitaria se han configurado como un problema de salud pública debido a las repercusiones individuales, sociales, económicas, legales y éticas que conlleva. Se ha descrito que todos los pacientes están expuestos a múltiples riesgos y uno de cada diez sufre algún tipo de lesión o daño durante la hospitalización ${ }^{(4,5)}$.

Los errores con medicamentos representan algunas de las fallas más frecuentes en la asistencia sanitaria ${ }^{(6)}$ y pueden causar complicaciones en el cuadro clínico, aumentar el tiempo de hospitalización e incluso generar discapacidad o muerte, la Organización Mundial de la Salud, reconociendo la importancia de la identificación, prevención y manejo de problemas y errores con medicamentos, dio a conocer en el año 2017 el tercer reto por la seguridad del paciente, Medication without harm, mediante el cual busca impulsar acciones en todos los niveles de la atención sanitaria que promuevan el uso seguro de medicamentos, limiten la exposición a riesgos y fomenten la calidad asistencial.

De acuerdo con el National Coordinating Council for Medication Error Reporting and Prevention (NCC MERP) los errores con medicamentos se pueden definir como cualquier incidente que pueda causar daño al paciente o dé lugar a la utilización inapropiada de los medicamentos, entendiendo que su utilización es una actividad rutinaria, habitual y multidisciplinaria que requiere conocimientos, destrezas y habilidades que garanticen el uso seguro durante todo el proceso, destacando que los errores pueden relacionarse con la práctica profesional, los procesos asistenciales y/o los sistemas sanitarios ${ }^{(7)}$.

EI NCC MERP clasifica los errores en nueve categorías denominadas con las letras del alfabeto desde la A hasta la I y organizadas en cuatro niveles de severidad: sin error, error sin daño, error con daño y error fatal. Se esquematiza en forma circular para demostrar la relación entre cada categoría y asignarle la misma proporción de área a cada una.

El área quirúrgica de los hospitales no es ajena a esta problemática, algunos autores resaltan que junto a las unidades de cuidado intensivo y salas de ginecoobstetricia son las que mayores riesgos presentan para sus usuarios, debido principalmente a la dinámica de trabajo que requiere altos niveles de atención, exigido por la condición clínica del paciente, cumplimiento de metas administrativas, uso constante de medicamentos considerados de alto riesgo (sedantes, opioides, vasoactivos y relajantes musculares), diferentes dispositivos biomédicos, entre otros ${ }^{(5,8)}$. Los anestesiólogos y el personal de enfermería están involucrados en el proceso de ordenar, despachar, administrar y hacer seguimiento a los efectos de estos medicamentos, mientras ejecutan otra serie de tareas en un ambiente de trabajo dinámico, por lo que los errores en los ambientes quirúrgicos no son $\operatorname{raros}^{(9,10)}$. Este ambiente dinámico y complejo puede crear condiciones favorables para cometer errores en la administración de medicamentos, especialmente en pacientes que requieren anestesia general durante el procedimiento 
quirúrgico ${ }^{(11,12)}$. Adicionalmente, el paciente, dependiendo de su condición clínica, debe ingresar a la sala de recuperación posanestésica, otra área de gran dinamismo y complejidad en la que se realiza el seguimiento a los efectos residuales de la anestesia y fármacos administrados en el intraoperatorio y de otros medicamentos utilizados para el manejo de signos y síntomas propios de cada intervención quirúrgica.

Diferentes autores destacan que en promedio un paciente hospitalizado está expuesto al menos a un error de medicación por día, relacionado con alguno de los pasos del proceso de administración de medicamentos $^{(13)}$, además se ha mostrado que el $80 \%$ de los errores con medicamentos hospitalarios son causados por error humano. En la misma línea se han descrito errores relacionados con la vía de administración, la subdosificación, la mezcla incorrecta de medicamentos, el almacenamiento y despacho inadecuado, los cambios inadvertidos de ampollas y jeringas, entre otros ${ }^{(9,14)}$. La National Patient Safety Agency (NPSA), entre 2004 y 2006, revisó más de 12.000 incidentes anestésicos, de los cuales 1,120 fueron errores de medicación y el $1,3 \%$ de los casos se asociaron con daño severo o muerte. De la misma forma, la Sociedad Japonesa de Anestesiólogos investigó 27.454 procedimientos de anestesia durante un período de 8 años (1999-2007) y, del total, 233 procedimientos se relacionaron con algún tipo de error de medicación, mostrando que, aunque infrecuentes, la mayor parte de ellos fueron claramente evitables ${ }^{(10)}$. Debido a la complejidad del proceso de utilización de medicamentos, existe una alta probabilidad de cometer errores ${ }^{(15)}$.

En las últimas décadas, en distintos lugares del mundo se han realizado esfuerzos organizados para conocer cuáles son los errores con medicamentos, identificando principalmente sus causas para facilitar la puesta en práctica de soluciones efectivas. Por tal razón, se propuso este estudio, con el objetivo de analizar los errores con medicamentos antes, durante y después de la administración de anestesia general para la realización de cirugía abdominal en un hospital de IV nivel en Bogotá, Colombia, como aporte al reto mundial, a la farmacovigilancia y, en general, al sistema de calidad en la atención y buscando promover la cultura de la seguridad a través de la identificación, reporte y clasificación de los errores con medicamentos que permitan guiar las decisiones necesarias para prevenirlos.

\section{METODOLOGÍA}

Se realizó un estudio observacional, descriptivo de corte transversal y que corresponde a un estudio de utilización de medicamentos sobre las consecuencias prácticas de su utilización. Esta metodología ha demostrado ser capaz de identificar mayores errores comparado con otros métodos como el autorreporte, reporte de incidencias, revisiones de historias clínicas entre otras.

Se incluyeron los procedimientos quirúrgicos abdominales (programados o de urgencia) de pacientes mayores de 18 años durante los últimos seis meses del año 2019 y en quienes estuvo indicada la administración de anestesia general. Se excluyeron las cirugías de pacientes que requirieron traslado posoperatorio inmediato a la unidad de cuidado intensivo. Los participantes fueron entrevistados a través de un cuestionario estructurado para obtener información sobre variables sociodemográficas, antecedentes farmacológicos, entre otros. Posteriormente se observó el proceso de utilización de medicamentos durante el pre e intra operatorio y posoperatorio inmediato en sala de recuperación

Se calculó el tamaño de muestra teniendo en cuenta una prevalencia de errores de medicación del $50 \%$ y una error alfa del $5 \%$ utilizando la fórmula para estudios descriptivos $^{(16)}$. De un total de 897 cirugías (245 programados y 652 de urgencia) se obtuvo un tamaño de muestra de 390 (149 programada y 241 de urgencia), las cuales fueron seleccionadas por el método de selección sistemática de elementos muestrales ${ }^{(17)}$.

Se observó la utilización de medicamentos en los tres turnos laborales -mañana, 
tarde y noche- desde el preoperatorio hasta el postoperatorio inmediato. La categorización del error, tipo y causas se realizó de acuerdo con la taxonomía establecida por la NCC MERP. Se utilizó la hoja de cálculo de Microsoft Excel ${ }^{\circledR}$ para estimar las medianas, promedio y desviación estándar, o proporciones según fuera el caso. Para determinar el nivel de riesgo de gravedad de las potenciales interacciones se revisó el programa Drug Interactions Checker ${ }^{(18)}$. Los aspectos relacionados con estabilidad fisicoquímica fueron documentados a partir de revisión en la página web de Stabilis ${ }^{\circledR(19)}$. Los medicamentos fueron clasificados de acuerdo con la categoría Anatomical Therapeutic Chemical Classification (ATC) ${ }^{(20)}$.

Esta investigación se considera con riesgo mínimo (Resolución No. 008430 del Ministerio de Salud, del 4 de octubre de 1993, Artículo 11). A pesar de esto, se solicitó el consentimiento informado de los usuarios y/o sus responsables, previo al inicio de la aplicación del instrumento de recolección de datos, se explicaron y aclararon las dudas e inquietudes surgidas, respetando la decisión final de los posibles participantes. Para mitigar posibles daños al paciente, luego de la identificación de un error con medicamentos, el responsable de la recolección de datos le comentó al responsable de la administración del medicamento el error en el que estaba incurriendo antes de que el medicamento llegara al paciente. El protocolo del estudio fue aprobado por el comité de ética institucional bajo el código DVO005 572-CV1002, el 10 de enero de 2019.

\section{RESULTADOS}

\section{Características sociodemográficas y clínicas}

Se observó el proceso de utilización de medicamentos en la totalidad de la muestra estimada (390 pacientes). Las características sociodemográficas reflejaron que el 60,25\% $(n=235)$ de la muestra pertenece al sexo femenino, de acuerdo con la distribución por edades se identificó que el $64,1 \% \quad(n=250)$ era menor de 70 años, la mediana fue de 54 años, con rango 18-90 años de edad.

El $62,8 \%(245 / 390)$ de los pacientes presentaba al menos una enfermedad de base, entre las que se destacaron las enfermedades crónicas no transmisibles como la hipertensión arterial 52,4\% (128/245), seguida por hipotiroidismo $16,3 \%(40 / 245)$, diabetes mellitus tipo II 13,5\% (33/245), cáncer 7,3\% (18/245), enfermedad pulmonar obstructiva crónica $5,3 \%(13 / 245)$, artritis $2,8 \%(7 / 245)$, y otras patologías 2,4\% (6/245). Del total de pacientes con patologías de base el $83,2 \%(204 / 245)$ refirió consumir algún tipo de medicamento. De acuerdo con la categoría ATC, los medicamentos más consumidos por estos pacientes correspondieron a la categoría C (fármacos cardiovasculares) con un 86,2\% (176/204), seguido por la categoría H03 (fármacos para terapia tiroidea) con un 26,4\% (54/204), y la categoría A10 (fármacos antidiabéticos) con un $18,6 \%(38 / 204)$, agentes para alteraciones de la vía respiratoria 4,4\% (9/204), otras categorías 3,9\% (8/204). Las alergias farmacológicas fueron referidas en el 6,9\% (27/390) de los pacientes, relacionadas principalmente con la penicilina, dipirona, algunos opioides y antiinflamatorios no esteroides (AINE).

El mayor número de procedimientos (programados y urgencias) se realizó en el turno de la tarde $39 \%$ (152/390), las cirugías programadas tuvieron una distribución similar entre el turno de la mañana 49,6\% (74/149) y la tarde $48,3 \%(72 / 149)$ mientras que el mayor número de urgencias se realizó durante el turno de la noche $44,3 \%$ (107/241). Los procedimientos quirúrgicos realizados fueron principalmente herniorrafías con un 26,4\% (103/390) y colecistectomías por laparoscopia $22,5 \%$ (88/390), seguido por laparotomías exploratorias 20,5\% (80/390), apendicetomías $18,9 \%$ (74/390), y otras $11,5 \%$ (45/390).

\section{Identificación de errores de medicación}

Durante el periodo de observación se registraron 3.677 administraciones de medicamentos, de las cuales el 59,6\% (2.190/3.677) presentaron por lo menos un error. El 69,4\% 
(1.520/2.190) de estos errores se identificaron en el pre e intraoperatorio y el $30,5 \%$ (670/2.190) en el posoperatorio inmediato. Las cirugías de urgencia aportaron un 59,6\% de los errores (907/1.520) mientras que las cirugías programadas un 40,3\% (613/1.520). El turno de la noche aportó la mayor cantidad de errores con 38,9\% (852/2.190), seguido por la tarde con $36,9 \%(810 / 2.190)$ y la mañana con $24,1 \%$ (528/2.190). En el intraoperatorio se presentan dos momentos relevantes: inducción de anestesia y transoperatorio. Las características generales de los errores identificados se presentan en la Tabla 1.

Durante el periodo postoperatorio se identificaron 670 errores. La Tabla 2 muestra que la mayor frecuencia se relacionó con la no utilización de elementos de protección personal seguido del no lavado de manos y la reutilización de medicamentos. No se identificaron errores en los 5 correctos (administración, paciente, medicamento, dosis y vía).

Tabla 1. Clasificación de errores con medicamentos en cirugía programada y cirugía de urgencia según períodos preoperatorio e intraoperatorio. Bogotá, Colombia, enero a septiembre, 2019.

\begin{tabular}{|c|c|c|c|c|c|}
\hline \multirow{2}{*}{$\begin{array}{l}\text { Tipo de error } \\
\text { Preoperatorio }\end{array}$} & \multirow[t]{2}{*}{$\begin{array}{l}\text { Categoría } \\
\text { del error }{ }^{1}\end{array}$} & \multicolumn{2}{|c|}{$\begin{array}{c}\text { Cirugía } \\
\text { programada }\end{array}$} & \multicolumn{2}{|c|}{$\begin{array}{l}\text { Cirugía de } \\
\text { urgencia }\end{array}$} \\
\hline & & $\mathrm{N}$ & $\%$ & $\mathrm{~N}$ & $\%$ \\
\hline Error en la hora & B & 2 & 0,3 & 104 & 11,5 \\
\hline No lavado de manos antes de diluir medicamento & A & 14 & 2,3 & 36 & 4,0 \\
\hline No utiliza elementos de protección personal & $A$ & 4 & 0,7 & 0 & - \\
\hline No realiza doble chequeo del medicamento antes de administrarlo & $C$ & 19 & 3,1 & 19 & 2,1 \\
\hline \multicolumn{6}{|l|}{ Intraoperatorio - Inducción de anestesia } \\
\hline No lavado de manos antes de diluir medicamento & A & 13 & 2,1 & 34 & 3,7 \\
\hline No limpieza de tapa elastomérica de viales & A & 65 & 10,6 & 125 & 13,8 \\
\hline Error en etiquetado de medicamentos diluidos & $\mathrm{C}$ & 67 & 10,9 & 84 & 9,3 \\
\hline Reutilización de medicamentos diluidos, en diferentes pacientes & $\mathrm{C}$ & 45 & 7,3 & 86 & 9,5 \\
\hline Reutilización de remanente de medicamentos en diferentes pacientes & $\mathrm{C}$ & 50 & 8,2 & 56 & 6,2 \\
\hline No confirmación de administración de medicamento & $A$ & 35 & 5,7 & 30 & 3,3 \\
\hline Error al programar bomba de infusión & B & 16 & 2,6 & 4 & 0,4 \\
\hline \multicolumn{6}{|l|}{ Intraoperatorio - Transoperatorio } \\
\hline No utiliza elementos de protección personal & A & 24 & 3,9 & 13 & 1,4 \\
\hline No realiza doble chequeo del medicamento antes de administrarlo & $A$ & 27 & 4,4 & 18 & 2,0 \\
\hline Error en etiquetado de medicamentos diluidos & $\mathrm{C}$ & 45 & 7,3 & 66 & 7,3 \\
\hline Reutilización de medicamentos diluidos, en diferentes pacientes & $\mathrm{C}$ & 22 & 3,6 & 37 & 4,1 \\
\hline Reutilización de remanente de medicamentos en diferentes pacientes & $\mathrm{C}$ & 12 & 2,0 & 11 & 1,2 \\
\hline Error al programar bomba de infusión & B & 4 & 0,7 & 4 & 0,4 \\
\hline Diluye varios medicamentos en la misma solución & $C$ & 24 & 3,9 & 34 & 3,7 \\
\hline No descarta medicamentos sobrantes & A & 113 & 18,4 & 137 & 15,1 \\
\hline No se irriga el equipo de infusión después de administrar el medicamento & $\mathrm{C}$ & 5 & 0,8 & 0 & - \\
\hline No registra en historia clínica & B & 7 & 1,1 & 9 & 1,0 \\
\hline TOTAL & & 613 & 100,0 & 907 & 100,0 \\
\hline
\end{tabular}

Fuente: Elaboración propia

'Según categorías de la National Coordinating Council for Medication Error Reporting and Prevention (NCC MERP). 
Tabla 2. Clasificación de errores con medicamentos en el posoperatorio. Bogotá, Colombia, enero a septiembre, 2019.

\begin{tabular}{l} 
Tipo de error \\
No lavado de manos antes de diluir medicamento \\
Farmacia despacha medicamento incorrecto \\
No utiliza elementos de protección personal \\
No realiza doble chequeo del medicamento antes de administrarlo \\
Error en etiquetado de medicamentos diluidos \\
\hline Reutiliza medicamentos diluidos en otro procedimiento \\
\hline Hora incorrecta \\
Reutiliza remanente de medicamentos en multidosis \\
Diluye varios medicamentos en la misma solución \\
\hline No registro en historia clínica \\
Total
\end{tabular}

\begin{tabular}{crr}
$\begin{array}{c}\text { Categoría } \\
\text { del error }\end{array}$ & $\mathrm{n}$ & $\%$ \\
$\mathrm{~A}$ & 124 & 18,5 \\
$\mathrm{~B}$ & 6 & 1,0 \\
$\mathrm{~A}$ & 153 & 22,8 \\
\hline $\mathrm{C}$ & 20 & 3,0 \\
\hline $\mathrm{C}$ & 114 & 17,0 \\
\hline $\mathrm{C}$ & 92 & 13,7 \\
\hline $\mathrm{C}$ & 45 & 7,5 \\
\hline $\mathrm{C}$ & 7 & 1,0 \\
C & 10 & 1,5 \\
A & 99 & 14,0 \\
\hline & 670 & 100,0
\end{tabular}

Fuente: Elaboración propia.

${ }^{1}$ Según categorías de la National Coordinating Council for Medication Error Reporting and Prevention (NCC MERP).

Los errores identificados en los periodos intra y postoperatorio, asociados a mayor severidad, incluyeron la no rotulación o rotulación inadecuada de medicamentos diluidos. Durante la inducción de anestesia los medicamentos involucrados fueron propofol 30,3\% (61/201) seguido del cisatracurio con un
$17,9 \%(36 / 201)$, fentanil con $17,4 \%(35 / 201)$, rocuronio con $10,9 \%$ (22/201), lidocaína $8,5 \%$ (17/201), midazolam 7,5\% (15/201), succinilcolina $4 \%(8 / 201)$ y ketamina $3,5 \%$ (7/201). En el transoperatorio se identificó la dipirona $29,1 \%$ (38/131), hidromorfona $16,8 \%$ (22/131), tramadol $16,8 \%$ (22/231),

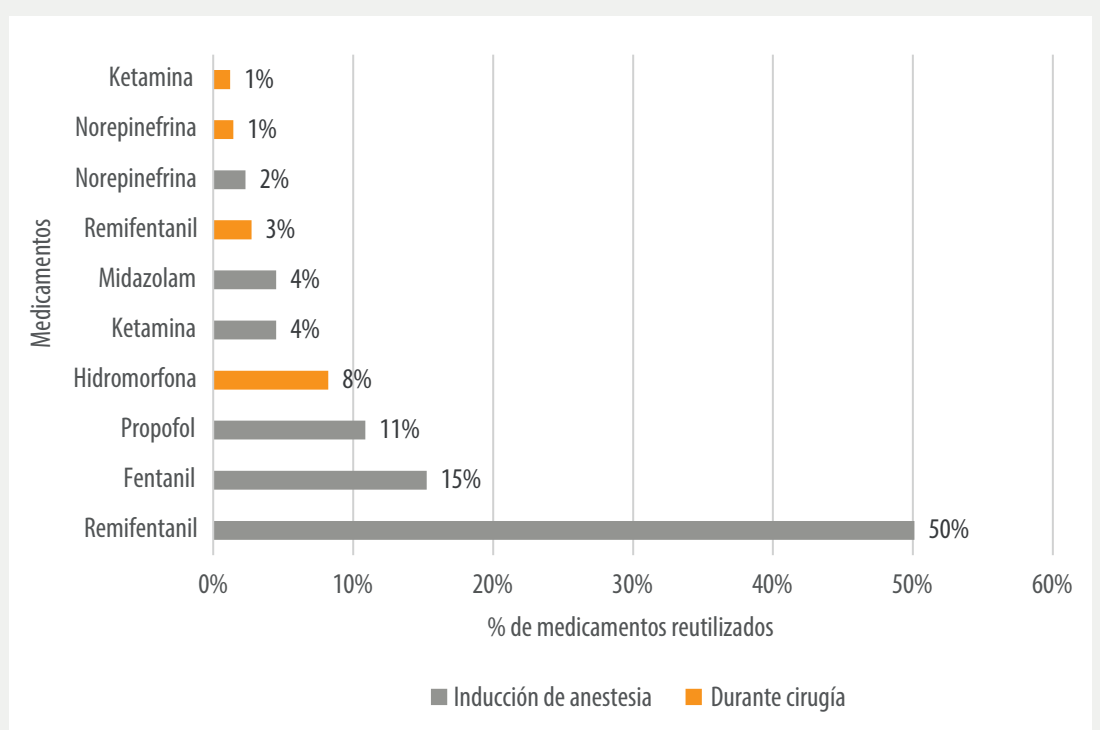

Figura 1. Medicamentos reutilizados en el período intraoperatorio (inducción de anestesia y durante la cirugía). Bogotá, Colombia, enero a septiembre, 2019.

Fuente: Elaboración propia. 


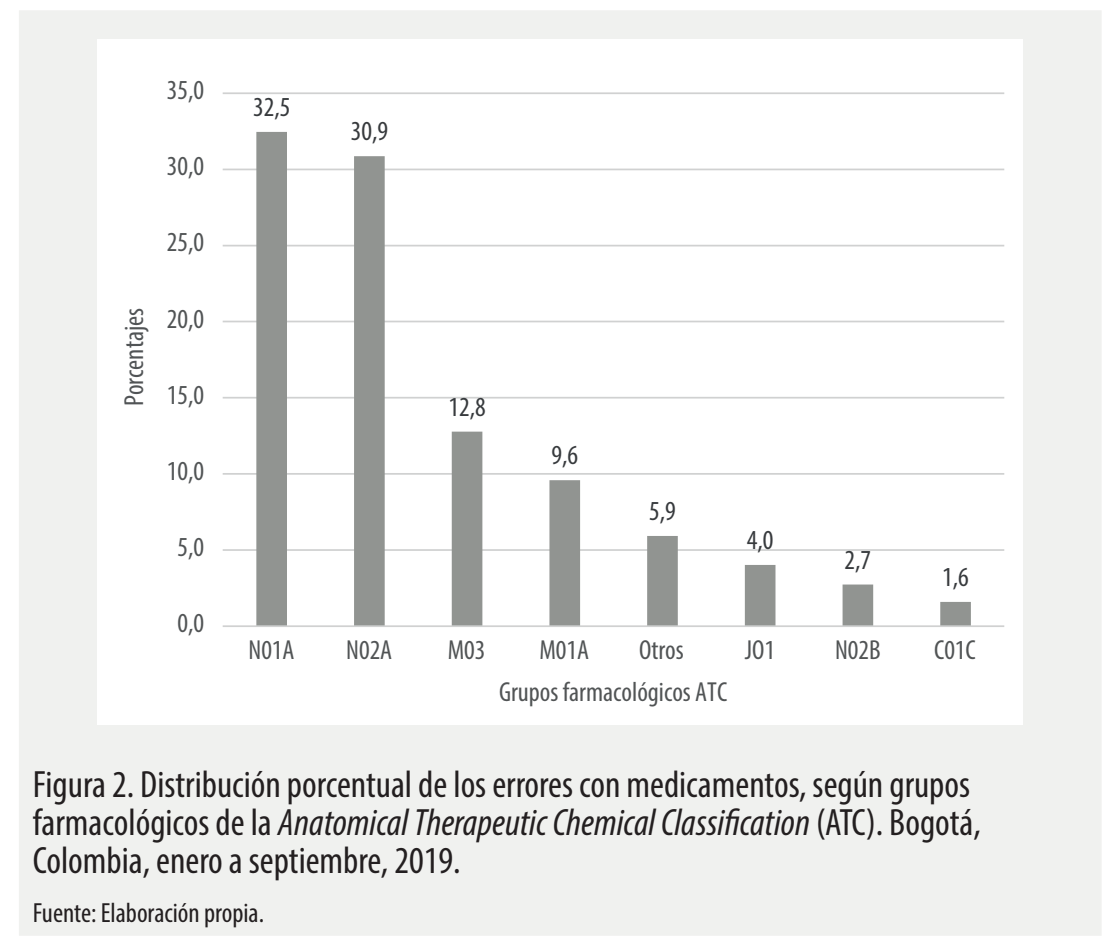

diclofenaco $15,3 \% \quad(20 / 131)$, norepinefrina $11,5 \%(15 / 131)$, ranitidina $6,1 \%(8 / 131)$ y metoclopramida $4,6 \%(6 / 131)$, mientras que en la sala de recuperación posanestésica los opioides, como hidromorfona 93,9\% (107/114), morfina $3,5 \%$ (4/114) y meperidina $2,6 \%$ (3/114) fueron los medicamentos que más estuvieron relacionados con este tipo de error.

Se reutilizaron en total 418 medicamentos, principalmente, durante la inducción de anestesia con un 56,7\% (237/418), seguido por el periodo postoperatorio 23,7\% (99/418) y el transoperatorio 19,6\% (82/418). La Figura 1 muestra el remifentanil como el medicamento más relacionado con este error, mientras que en el transoperatorio fue la hidromorfona, al igual que en el postoperatorio inmediato.

La dilución de dos o más medicamentos en una misma solución se observó en 68 oportunidades, siendo la dipirona el medicamento más relacionado con esta práctica, la cual fue mezclada, entre otros, con diclofenaco 14,7\% $(10 / 68)$, ranitidina $13,2 \%$ (9/68), tramadol $11,7 \%(8 / 68)$ y metoclopramida $11,7 \%(8 / 68)$.
Otras mezclas en la que no se involucró la dipirona fueron con ranitidina, tramadol, hioscina 48,5\% (33/68).

Los grupos farmacológicos asociados a algún tipo de error fueron principalmente el N01A con un 32,5\% (711/2.190), seguidos del N02A con un 30,8\% (676/2.190) y el M03 con un 12,8\% (280/2.190). La Figura 2 describe la frecuencia de errores.

\section{DISCUSIÓN DE RESULTADOS}

De acuerdo con la distribución demográfica presentada, se identificó una población con características similares a las reportadas en otros estudios $^{(21,22)}$ que describen, principalmente, una población de pacientes jóvenes, en edad productiva, pertenecientes en mayor proporción al sexo femenino.

Más de la mitad de los pacientes observados presentó al menos una patología de base, principalmente, enfermedades crónicas no transmisibles que, en la mayoría de los 
casos, no presentaron relación directa con el motivo de la intervención quirúrgica. Sin embargo, se resalta que este tipo de enfermedades se asocian al consumo habitual y concomitante de dos o más medicamentos, lo cual, de acuerdo con la literatura está relacionado con mayor número de errores, reacciones adversas, interacciones farmacológicas, entre otros ${ }^{(23,24)}$.

Previo al acto anestésico, siempre se tuvieron en cuenta los antecedentes patológicos y farmacológicos sin identificar posibles interacciones farmacodinámicas ni farmacocinéticas entre el medicamento de uso frecuente del paciente y los requeridos para la inducción y mantenimiento de la anestesia ${ }^{(25,26)}$.

Dentro de los antecedentes farmacológicos, otro aspecto importante son las alergias conocidas a medicamentos, las cuales se constituyen como un riesgo para el paciente, en este estudio se reportó un reducido porcentaje de alergia a algún medicamento, lo cual es esperable ya que esta condición se presenta en un escaso número de personas $^{(27)}$. No se identificaron errores asociados con esta variable, lo que puede suponer una fortaleza del programa de farmacovigilancia institucional y las estrategias implementadas para la prevención de errores con medicamentos en este grupo de pacientes, principalmente el uso de manilla de identificación de un color especifico.

\section{Preoperatorio}

De acuerdo con diferentes autores los procedimientos quirúrgicos de urgencia comparados con los electivos o programados tienden a presentar un mayor número de complicaciones tanto intraoperatorias como posoperatorias $^{(28)}$, asociadas en ocasiones a errores en la administración de medicamentos. Los resultados identificados reflejan este aspecto y coinciden con Abeysekera et al. ${ }^{(29)}$, quienes reportan que más del $50 \%$ de los errores son documentados en cirugías de urgencia, asimismo reportaron que aproximadamente el $10 \%$ de estos se configuraron en lesión o daños, lo que difiere del presente estudio, pudiendo estar determinado por el tiempo de observación y número de casos captados. Este aspecto llama la atención ya que puede indicar que existen riesgos latentes que no han sido intervenidos, siendo susceptibles de configurarse en eventos adversos.

Durante el proceso de preparación prequirúrgica de todo paciente que ingresa al servicio de salas de cirugía, uno de los aspectos de mayor relevancia es la administración de profilaxis antibiótica ${ }^{(30)}$, la cual fue administrada a la totalidad de pacientes de la muestra. Sin embargo, un poco menos de la cuarta parte de los pacientes no recibió el medicamento entre 30 y 60 minutos antes de la incisión quirúrgica, de acuerdo con la recomendación dada en diferentes guías y estudios internacionales ${ }^{(31,32,33)}$, hallando similitud con lo reportado por Gutiérrez et al. ${ }^{(34)}$, quienes identificaron que en el $12 \%$ de los casos se presentó este error, exponiendo al paciente a múltiples riesgos, como generar o exacerbar reacciones adversas propias de cada medicamento y/o interacciones farmacológicas al administrar el antibiótico en bolo y en concomitancia con agentes hipnóticos, sedantes, opioides y relajantes musculares durante la inducción de anestesia, además del riesgo aumentado de desarrollar infección del sitio operatorio al no alcanzar concentraciones óptimas del fármaco en los tejidos antes de ser expuestos a la incisión quirúrgica ${ }^{(34,35,36,37)}$.

Este error se presentó en mayor proporción durante el turno nocturno, coincidiendo con algunas investigaciones nacionales que asocian un mayor número de errores y eventos adversos a este turno ${ }^{(38,39)}$ posiblemente por el menor número de personal asistencial, el agotamiento físico y mental y, específicamente, en algunos casos, por la no formulación del antibiótico, impidiendo que la farmacia lo despache. Es importante mencionar que la aplicación de la lista de chequeo de seguridad de la cirugía permitió identificar la totalidad de los casos de no administración del medicamento.

La doble verificación antes de administrar medicamentos es una recomendación descrita en la bibliografía, especialmente con los medicamentos utilizados por vías parenterales, 
la experiencia del National Health System (NHS), en Inglaterra, refleja resultados positivos en la prevención de errores asociados a medicamentos. La no realización de este paso puede dar lugar a la progresión de posibles errores incluso con medicamentos catalogados como de riesgo intermedio o bajo ${ }^{(40)}$.

\section{Intraoperatorio}

De acuerdo con los resultados, el periodo intraoperatorio está relacionado con un mayor número de errores, comparado con los otros dos periodos que comprenden el perioperatorio, esto puede estar asociado a las características propias de la administración de medicamentos en el quirófano que incluyen multiplicidad de tareas en un mismo momento, mayor número de órdenes verbales y mayor uso de medicamentos diluidos en diferentes jeringas. Además, en algunos casos, puede existir deficiencia en la comunicación entre el equipo de profesionales sanitarios ${ }^{(41)}$, generando factores ideales para la incidencia y prevalencia de errores.

Uno de los aspectos de mayor relevancia, previo a la dilución y administración de medicamentos, es el lavado de manos. Desde 2005, la Organización Mundial de la Salud ha insistido en esta actividad como una intervención primaria para garantizar una atención limpia y más segura, actualmente la NCC MERP reconoce que no lavarse las manos previo a la dilución y administración de medicamentos puede considerarse como un error en la utilización segura de estos, especialmente, cuando se manipulan medicamentos que serán administrados por vías parenterales $^{(42)}$.

En este estudio se encontró que aproximadamente en 1 de cada 10 casos no se hizo lavado ni higienización de manos previo a la administración de medicamentos para la inducción de anestesia, se debe tener en cuenta que, durante el proceso de atención quirúrgica, el personal asistencial está en contacto frecuente con el entorno y fluidos del paciente, además de manipular directamente equipos de infusión, Ilaves de tres vías, jeringas y otros dispositivos médicos para administrar medicamentos, esta información, aunque en menor medida, es consistente con lo reportado por Rowlands et al. ${ }^{(43)}$ quienes demuestran que la inducción de anestesia es uno de los momentos en los que menos se cumple el lavado de manos y los elementos para administrar medicamentos se incluyen dentro de los 20 objetos que más se tocan con las manos sin lavar dentro del quirófano, promoviendo posibles infecciones bacterianas, virales y micóticas.

Por otro lado, la rotulación adecuada de los medicamentos diluidos en jeringas o soluciones es fundamental para prevenir errores, principalmente, en ambientes tan dinámicos como el quirófano en los que suelen utilizarse diferentes medicamentos con índice terapéutico estrecho ${ }^{(44)}$. En la práctica clínica diaria se evidencian diferentes estrategias para evitar la administración de un medicamento que no se requiere o que se necesita en dosis diferentes, como la utilización de jeringas de diferentes tamaños, la rotulación con tinta indeleble o simplemente dibujar una letra o señal en la jeringa. No obstante, ninguna de estas prácticas tiene un claro respaldo científico por lo que, en la mayoría de las ocasiones, se las califica como insuficientes a la hora de prevenir errores ${ }^{(45)}$. En este estudio, se identificó que durante todos los turnos se presentaron casos de no rotulación de medicamentos diluidos, especialmente, en jeringas utilizadas durante la inducción de anestesia, soluciones de remifentanil y jeringas con medicamentos diluidos como hidromorfona y morfina. Esta tendencia fue mucho más marcada en el turno de la noche y aunque no se evidenció ningún evento adverso inmediato, es claro que esta actividad va en contravía de lo recomendado por organizaciones nacionales e internacionales y acuerdos por la seguridad del paciente ${ }^{(46)}$.

Estos resultados son congruentes con reportes de otras publicaciones ${ }^{(45,47)} y$, aunque proporcionalmente son pocos los casos, la no rotulación de jeringas y soluciones puede traer consecuencias importantes en la integridad del paciente. Además, permiten inferir que es un error común en el área de 
quirófanos y la única barrera entre el error y el paciente es la pericia o experticia de quien administra el medicamento.

Durante el proceso intraoperatorio, uno de los errores con mayor proporción de ocurrencia es la reutilización de medicamentos diluidos y administrados en pacientes diferentes. Esta práctica limita la posibilidad de garantizar la esterilidad de los medicamentos para administración intravenosa y aumenta el riesgo de contaminación no solo del medicamento sino también del envase o dispositivo utilizado para administrarlo, convirtiendo las jeringas, equipos de infusión y bolsas de solución en vectores mecánicos potenciales para la transmisión de patógenos entre pacientes ${ }^{(48,49,50,51,52)}$.

Los medicamentos que se reutilizaron en mayor proporción durante la inducción de anestesia fueron remifentanil, fentanil y propofol, lo cual difiere con lo reportado por otros autores quienes describen al propofol como el principal medicamento involucrado en este error ${ }^{(51,53)}$. Esta diferencia puede estar determinada por el mayor número de reportes de contaminación y transmisión de infecciones que hay frente a este medicamento a nivel mundial ${ }^{(54,55,56)}$. Respecto al remifentanil, su reutilización está relacionada con la dilución en soluciones de hasta $500 \mathrm{cc}$, pudiendo ser utilizada durante toda una jornada de trabajo (6 horas en el día y 12 horas en la noche) y, por tanto, administrada a más de 1 paciente, incumpliendo con recomendaciones de los fabricantes, quienes destacan que la estabilidad fisicoquímica del medicamento diluido es hasta 4 horas en ambientes controlados ${ }^{(57)}$.

En el desarrollo del procedimiento quirúrgico varían los medicamentos involucrados en este error, se identificaron la hidromorfona, remifentanil y norepinefrina como los más reutilizados luego de ser diluidos. De la misma forma, la ketamina fue el medicamento del que más se tomaron dosis del mismo vial para diferentes pacientes pasando por alto técnicas de asepsia, antisepsia y administración segura de medicamentos. Todos estos fármacos fueron susceptibles de reutilizarse en por lo menos tres pacientes diferentes durante todos los turnos de trabajo y de forma independiente a si el procedimiento era programado o de urgencia, actuando en contravía de recomendaciones dadas por diferentes organismos internacionales ${ }^{(49,58)}$ que actualmente promueven la política institucional 1-1-1: 1 paciente, 1 medicamento, 1 insumo.

El error descrito guarda relación inversa con la baja proporción de descarte o segregación de los viales y dispositivos biomédicos con remanente de medicamento indicados para uso único, estudios como el de Kishi et al. ${ }^{(59)}$ muestran resultados contrarios a los haIlados en esta investigación, con un alto porcentaje de descarte de medicamentos, este hecho puede demostrar una baja adherencia a protocolos y recomendaciones internacionales, nacionales e institucionales sobre administración segura de medicamentos.

Por otro lado, las bombas de infusión constituyen una herramienta vital al momento de administrar algunos medicamentos, especialmente aquellos con índice terapéutico estrecho. Organizaciones como la Infusion Nurse Society (INS), la Sociedad Española de Infusión y Acceso Vascular (SEINAV), entre otras, indican que su uso adecuado se relaciona con la disminución de errores, como medicamento y dosis incorrecta, aunque también Ilaman la atención respecto de que una programación errónea y la omisión de alertas puede dar lugar a incidentes y eventos con resultados adversos como la muerte ${ }^{(60)}$.

Algunos estudios, han reportado que durante el uso de bombas de infusión identificaron una alta presencia de errores relacionados con su programación ${ }^{(61,62)}$, esta situación difiere de los hallazgos de esta investigación, posiblemente, por el carácter multicéntrico del estudio o el tipo de medicamentos administrados por bomba. En el presente estudio los medicamentos cuentan con un uso estandarizado y constante para el mantenimiento del estado anestésico e inotrópico principalmente, lo cual disminuye la variabilidad en los medicamentos y dosis que se deben programar. En todos los casos, se evitó que el error alcanzara al paciente cuando una segunda persona verificó la bomba de infusión.

Otro de los errores identificados tiene que ver con la limpieza de la tapa elastomérica 
o la superficie externa de los viales antes de ser utilizados, lo cual se reconoce como una práctica de buen uso de medicamentos ${ }^{(63)}$, algunas investigaciones han sugerido que se disminuye la carga bacteriana que puede colonizar estas zonas ${ }^{(64)}$; sin embargo, se observa que es una práctica poco realizada en quirófanos ${ }^{(65)}$. En este aspecto, nuestros resultados se correlacionan con los reportados por Kishi et al.(59), en los que no se realizó esta práctica en aproximadamente el $80 \%$ de los casos, promoviendo la posible contaminación del medicamento y la transmisión de agentes microbianos entre pacientes.

La mezcla de medicamentos en una misma solución es una práctica común al momento de administrarlos por vía intravenosa. Autores como Ruiz et al. ${ }^{(66)}$ recomiendan no hacer mezclas de más de tres medicamentos aun conociendo la compatibilidad entre estos. Por otro lado, Westbrook et al.(67) han reportado que no considerar la estabilidad y la compatibilidad de medicamentos es uno de los principales errores durante su administración por vía intravenosa. En este estudio se reportaron relativamente muy pocos casos de mezcla de medicamentos y en ninguno de ellos se identificaron reacciones adversas inmediatas. A pesar de esto, se debe tener en cuenta que el medicamento que más se utilizó durante esta práctica fue la dipirona, sobre la cual existen numerosos debates acerca de su seguridad, asociándola a efectos adversos graves como agranulocitosis y anafilaxia ${ }^{(68)}$. Por lo cual, es recomendable administrarla preferiblemente en unidosis. Aunque la práctica clínica diaria respalde la utilización de estas mezclas, no se dispone de datos concluyentes que aseguren que la estabilidad de las mezclas identificadas sea la adecuada(69,70,71).

\section{Posoperatorio}

Si bien, la sala de recuperación posanestésica es un área conexa con los quirófanos, debido a su dinámica, se asemeja más a un área de cuidado intensivo y hospitalización. A pesar de esto, los errores con medicamentos identificados fueron similares a los descritos durante el pre e intraoperatorio.

El no lavado o higienización de manos previo a la utilización de medicamentos es un error común a las dos áreas; sin embargo, la proporción de casos aumenta en más del doble en esta unidad, así como la baja adherencia al uso de elementos de protección personal como guantes y tapabocas, posiblemente, por una menor percepción de riesgo, mayor número de pacientes que demandan atención inmediata para el control de signos y síntomas ${ }^{(72)}$ o disposición inadecuada de elementos para realizar esta actividad, se debe destacar que el lavado de manos es una medida simple y económica relacionada con la prevención de infecciones asociadas al cuidado de la salud(73,74).

La no rotulación de los medicamentos diluidos y reutilización de jeringas también se consolidan como errores frecuentes en esta área, el medicamento que más se ve involucrado es la hidromorfona, debido principalmente a dos aspectos: 1) el control del dolor, y 2) la dilución del medicamento en jeringa permite contar con múltiples dosis de rescate analgésico que usualmente no son consumidas en su totalidad por un mismo paciente, dando la posibilidad de reutilizar la jeringa en varios individuos.

El análisis de la bibliografía demuestra que estos errores pueden ser comunes en algunos quirófanos y salas de recuperación posanestésica, en las que la adherencia a guías, recomendaciones y protocolos es baja, lo que representa un aumento en el riesgo de transmisión de infecciones; intercambio de jeringas; hurto de insumos y medicamentos; administración en horario, vía, dosis y paciente incorrectos; además de cuestionar la ética profesional de quienes incurren en esta práctica $^{(75,76,77,78)}$.

De la misma forma, la hidromorfona fue el medicamento que en menor frecuencia se registró en la historia clínica, especialmente, las dosis de rescate analgésico, la administración de medicamentos exige un estricto registro en la historia clínica del paciente, al ser este un documento legal, deben diligenciarse con veracidad los horarios, vías, 
complicaciones y eventos adversos que se hayan presentado durante el procedimiento, la ausencia de registro se asume como si no se hubiera administrado el medicamento ${ }^{(79)}$, facilitando la administración de dosis adicionales, administración de medicamentos que potencian o inhiben su respuesta conjunta, además de permitir un deficiente control en los viales e insumos utilizados.

\section{Clasificación de errores con medicamentos}

De acuerdo con la clasificación de los errores según la $\mathrm{NCC} M E R P^{(7)}$, los errores identificados en este estudio se enmarcaron en las tres primeras categorías, que representan errores potenciales y sin consecuencias clínicas relevantes para los pacientes. En diferentes revisiones de la bibliografía ${ }^{(72,80)}$ se ha encontrado que el mayor número de errores con medicamentos se encuentra en estas categorías. Como se mencionó, aunque no se configuran en lesión o daño, al prevalecer en el tiempo tienden a normalizarse e interiorizarse por parte del personal asistencial, calificándolos como inevitables e intrascendentes, pudiendo aumentar en frecuencia $y$, por tanto, aumentando el riesgo de desarroIlar resultados negativos.

Por otro lado, el estudio de Karthikeyan et al. ${ }^{(81)}$, realizado en un servicio de hospitalización, se asemeja al presente estudio en la metodología plantead. Sin embargo, los resultados difieren principalmente en la categoría C (error sin daño), posiblemente por el tipo de servicio estudiado, número del personal asistencial, giro cama, entre otros. Estudios como los de Machado et al. ${ }^{(82)}$ clasificaron los errores identificados en su análisis en la categoría A (circunstancias o eventos que tienen la capacidad de causar un error), como los más frecuentes en diferentes hospitales de Colombia, coincidiendo con los resultados del presente estudio y con lo descrito por Jornet-Montaña et al. ${ }^{(83)}$ quienes clasificaron aproximadamente el $90 \%$ de los errores como potenciales o no asociados a lesiones o daños para el paciente.
Por su parte, Grigg et al. ${ }^{(84)}$ también realizaron su estudio en quirófanos, describiendo el mayor número de errores como potenciales o sin daño para el paciente, sin embargo, en sus resultados si reportaron casos de error asociado a lesión, probablemente debido a un mayor tiempo de observación y mayor complejidad de los casos evaluados.

En una revisión de errores con medicamentos en anestesiología ${ }^{(85)}$ se determinó que los principales grupos farmacológicos asociados a errores son congruentes, en su mayoría, con los identificados en este estudio: opioides, anestésicos generales, antibióticos y vasoactivos. En la misma revisión se evidencia una comparación con los reportes de la base de datos MEDMARX ${ }^{(86)}$, y obtienen resultados muy similares a los de este estudio. Se destaca el caso de hidromorfona que, en MEDMARX, también se registra como uno de los medicamentos más asociado a errores, incluso con resultados fatales.

En este estudio no se identificaron errores que se pudieran clasificar en las categorías $\mathrm{E}$, $\mathrm{F}, \mathrm{G}$ o $\mathrm{H}^{(87)}$ relacionadas con la presencia de eventos adversos para el paciente, hallazgo que es similar no solo con los reportados en estudios recientes, sino también con los reportados hace más de una década y que sugieren que los errores, aunque no generen lesiones evidentes de forma inmediata, prevalecen en las instituciones prestadoras de servicios de salud y son multifactoriales e independientes del entorno de hospitalización. Además, en un momento determinado, pueden inducir resultados negativos para el paciente, el profesional sanitario y la institución.

A pesar de ser un estudio observacional, por razones éticas fue necesario advertir al responsable de la administración del medicamento sobre el error observado por el investigador, aspecto que seguramente influyó en los resultados. Dado que no estaba previsto en el estudio, no se cuantificaron las advertencias realizadas a los profesionales de la salud sobre el tipo de error intervenido. 


\section{CONCLUSIONES}

En más de la mitad de los casos de utilización de medicamentos se identificó por lo menos un error, clasificado de acuerdo con el NCC MERP en las categorías A, C y B, respectivamente. Los errores más frecuentes están relacionados con la falta de limpieza de la tapa elastomérica de los viales antes de diluir y/o administrar el medicamento, el no descarte de medicamentos sobrantes, la falta o inadecuada rotulación de jeringas y soluciones diluidas y la reutilización de jeringas y soluciones con diferentes pacientes.

El turno tarde fue el que aportó mayor número de errores durante el intraoperatorio mientras que el turno noche lo fue en la sala de recuperación posanestésica. El grupo farmacológico que se relacionó con mayor número de errores fueron los anestésicos generales, con el remifentanil, fentanil y propofol como los principales medicamentos involucrados.

Todos los errores identificados se definieron como situaciones con potencial de daño, que indican la necesidad de continuar la promoción de la estandarización de actividades durante la utilización de medicamentos y la cultura de seguridad del paciente. Se recomienda para futuros estudios, cuantificar las intervenciones realizadas durante la realización del estudio, así como el tipo de error que se evitó.

\section{REFERENCIAS BIBLIOGRÁFICAS}

1. Adams Pizarro I, Walker Z, Robinson J, Kelly S, Toth M. Using the AHRQ hospital survey on patient safety culture as an intervention tool for regional clinical improvement collaboratives. En: Henriksen K, Battles JB, Keyes MA, et al., eds. Advances in patient safety: new directions and alternative approaches (Vol. 2: Culture and Redesign). Rockville: Agency for Healthcare Research and Quality; 2008.

2. Aranaz Andrés J, Aibar Remón C. Estudio IBEAS: Prevalencia de efectos adversos en hospitales de Latinoamérica. Madrid: Ministerio de Sanidad, Política Social e Igualdad; 2010.

3. Kohn LT, Corrigan JM, Molla SD, eds. To err is human building a safer health system. Washington: National Academies Press; 2000.

4. Agência Nacional de Vigilância Sanitária. Assistência segura: uma reflexão teórica aplicada à prática [Internet]. Brasilia; 2017 [citado 10 oct 2020]. Disponible en: https://tinyurl.com/fbakk265.

5. Gaitán Duarte H, Eslava Schmalbach J, Rodríguez Malagon N, Forero Supelano V, Santofimio Sierra D, Altahona $\mathrm{H}$. Incidencia y evitabilidad de eventos adversos en pacientes hospitalizados en tres instituciones hospitalarias en Colombia, 2006. Revista de Salud Pública. 2008;10(2):215-226.

6. De los Ríos Bermúdez Y, Rodríguez Dulcey GB. Caracterización de los errores de medicación desde la perspectiva de los trabajadores de la salud de Empresas Sociales Del Estado de II nivel de atención del departamento del Tolima 2014. Medellín: Universidad de Antioquia, Universidad del Tolima; 2015.
7. National Coordinating Council for Medication Error Reporting and Prevention. About medication errors [Internet]. 2021 [citado 10 oct 2020]. Disponible en: https://tinyurl.com/ye6k3t95.

8. Kothari D, Gupta S, Sharma C, Kothari S. Medication error in anaesthesia and critical care: A cause for concern. Indian Journal of Anaesthesia. 2010;54(3):187192. doi: 10.4103/0019-5049.65351.

9. Berrío Valencia M. Prevención de errores en la administración de medicamentos en anestesia. Revista CES Medicina. 2014;28(2):307-312. doi: 10.21615/cesmed. v28i2.2925.

10. López Rabassa SI, Paz Estrada C, López Lazo S, González Rodríguez GS, Smith NR. Error relativo a medicamentos en anestesiología: ¿Cuál es la problemática? Revista Mexicana de Anestesiología. 2012;35(4):275-282.

11. Litman RS. How to prevent medication errors in the operating room? Take away the human factor. British Journal of Anaesthesia. 2018;120(3):438-440. doi: 10.1016/j.bja.2018.01.005.

12. Miranda JD, Vallejos L, Ñañez DP, Calvache JA. Errores en la administración de medicamentos intravenosos en pacientes sometidos a anestesia en el quirófano. Revista de la Facultad de Ciencias de la Salud Universidad del Cauca. 2013;15(1):23-28.

13. Choo J, Hutchinson A, Bucknall T. Nurses' role in medication safety. Nursing. 2010;18(7):853-861. doi: 10.1111/j.1365-2834.2010.01164.x

14. Esper C, Ra J, Carrillo LD. Incidentes críticos y error en la atención medica: ¿es el médico o el sistema? Medicina Interna de México. 2011;27(3):284-293. 
15. Pape TM. Applying airline safety practices to medication administration. Medsurg Nursing. 2003;12(2):77-94 .

16. Aguilar Barojas S. Fórmulas para el cálculo de la muestra en investigaciones de salud. Salud en Tabasco. 2005; 11(1-2):333-338.

17. Corral Y, Corral I, Franco Corral A. Procedimientos de muestreo. Revista Ciencias de la Educación. 2015;26(46):151-167.

18. Drug Interactions Checker. Drugs.com [Internet]. 2019 [citado 10 oct 2020]. Disponible en: https://tinyurl. com/5dmxpn2r.

19. Jean V, Rota JB, Gindre I, Daouphars M, Demoré B Georget S. Estabilidad y compatibilidad de los medicamentos [Internet]. 2019 [citado 10 oct 2020]. Disponible en: https://tinyurl.com/2sthtc4h.

20. World Health Organization. Anatomical Therapeutic Chemical (ATC) Classification [Internet]. 2019 [citado 10 oct 2020]. Disponible en: https://tinyurl.com/3yzxssv5.

21. Nanji KC, Patel A, Shaikh S, Seger DL, Bates DW. Evaluation of perioperative medication errors and adverse drug events. Anesthesiology. 2015;124(1):25-34 doi: 10.1097/ALN.0000000000000904.

22. Merry AF, Webster CS, Hannam J, Mitchell SJ, Henderson R, Reid P, Edwards KE, Jardim A, Pak N, Cooper J Hopley L, Frampton C, Short TG. Multimodal system designed to reduce errors in recording and administration of drugs in anaesthesia: prospective randomised clinical evaluation. BMJ. 2011;343:d5543. doi: 10.1136/bmj. d5543.

23. Rodríguez JY, Aguilar Armas J, Arana Delgado JC. Reacciones adversas medicamentosas durante el acto anestésico en pacientes sometidos a cirugía electiva en el centro quirúrgico del Hospital Almanzor Aguinaga Asenjo de octubre 2008 - marzo 2009. Revista del Cuerpo Médico del Hospital Nacional Almanzor Aguinaga Asenjo. 2013;6(3):9-17.

24. Soares Rodrigues MC, Oliveira C de. Interacciones medicamentosas y reacciones adversas a los medicamentos en polifarmacia en adultos mayores: una revisión integradora. Revista Latino-Americana de Enfermagem. 2016;24:e2800. doi: 10.1590/1518-8345.1316.2800.

25. Tafur Betancourt LA. El mundo oculto de las interacciones farmacológicas en anestesia. Revista Colombiana de Anestesiología. 2017;5(3):216-223. doi: 10.1016/j. rca.2017.05.003.

26. Berg JPVD, Vereecke HEM, Proost JH, Eleveld DJ, Wietasch JKG, Absalom AR, Struys MMRF. Pharmacokinetic and pharmacodynamic interactions in anaesthesia: A review of current knowledge and how it can be used to optimize anaesthetic drug administration. British Journal of Anaesthesia. 2017;118(1):44-57. doi: 10.1093/bja/aew312.

27. Jarernsiripornkul N, Chaipichit N, Chumworathayi $\mathrm{P}$, Krska J. Management for improving patients' knowledge and understanding about drug allergy. Pharmacy Practice. 2015;13(1):513. doi: 10.18549/PharmPract.2015.01.513.
28. Espinoza GR, Espinoza G JP. Calidad en cirugía: hacia una mejor comprensión de las complicaciones quirúrgicas. Revista Médica de Chile. 2016;144(6):752757. doi: 10.4067/S0034-98872016000600010.

29. Abeysekera A, Bergman IJ, Kluger MT, Short TG Drug error in anaesthetic practice: a review of 896 reports from the Australian Incident Monitoring Study database. Anaesthesia. 2005;60:220-227. doi: 10.1111/j.13652044.2005.04123.x.

30. Andersen B, Kallehave F, Andersen H. Antibiotics versus placebo for prevention of postoperative infection after appendicectomy. Cochrane Database of Systematic Reviews. 2003;(2):CD001439. doi: 10.1002/14651858. CD001439.

31. Ban AK, Minei JP, Laronga C, Harbrecht BG, Jensen EH, Fry DE, Itani KMF, Dellinger EP, Ko CY, Duane TM. American College of Surgeons and Surgical Infection Society: Surgical Site Infection Guidelines, 2016 Update. Journal of the American College of Surgeons. 2016;224(1):59-74. doi: 10.1016/j.jamcollsurg.2016.10.029.

32. Enzler MJ, Berbari E, Osmon DR. Antimicrobial prophylaxis in adults. Mayo Clinic Proceedings. 2011;86(7): 686-701. doi: 10.4065/mcp.2011.0012.

33. Steinberg J, Braun B, Hellinger W, Kusek L, Bozikis $M$, Bush A, Dellinger EP, Burke JP, Simmons B, Kritchevsky SB, Trial to Reduce Antimicrobial Prophylaxis Errors (TRAPE) Study Group. Timing of antimicrobial prophylaxis and the risk of surgical site infections: results from the trial to reduce antimicrobial prophylaxis errors. Annals of Surgery. 2009;250(1):10-16. doi: 10.1097/ SLA.0b013e3181ad5fca.

34. Gutiérrez DA, López JJ, Acero E, Hernández OE. Utilización profiláctica de antibióticos en la unidad médico-quirúrgica de un hospital de la ciudad de Bogotá. Revista Colombiana de Ciencias Químico Farmacéuticas. 2010;39(1):30-41. doi: 10.15446/rcciquifa.

35. Classen DC, Evans RS, Pestotnik SL, Horn SD, Menlove $\mathrm{RL}$, Burke JP. The timing of prophylactic administration of antibiotics and the risk of surgical-wound infection. New England Journal of Medicine. 1992;326(5):281286. doi: 10.1056/NEJM199201303260501.

36. Anderson DJ, Podgorny K, Berríos Torres SI, Bratzler DW, Dellinger EP, Greene L, Nyquist AC, Saiman L, Yokoe DS, Maragakis LL, Kaye KS. Strategies to prevent surgical site infections in acute care hospitals: 2014 update. infection control \& hospital epidemiology. Cambridge University Press. 2016;35(6):605-627. doi: 10.1086/676022.

37. Del Moral Luque JA, Colás Ruiz E, Gil Yonte P, Fernández Cebrián JM, Villardel Campo MC, Delgado Iribarren A, Valverde Cánovas JF, Rodríguez Caravaca G Evaluación de la adecuación de la profilaxis antibiótica en la cirugía de recto. Revista Española de Quimioterapia. 2017;30(1):14-18. doi: 10.37201/req/098.2019.

38. Saldaña DA, Colmenares SR, Álvarez JCD, Gómez J, Hernández AG, Díaz JE, Rodríguez SM, Rodríguez CE, Rodriguez AC. Caracterización de los eventos 
adversos reportados por enfermería en unidades de cuidado intensivo en Bogotá (Colombia). Salud Uninorte. 2017;33(2):105-117.

39. Alarcón Ruiz DJ, Gil Vargas LF. Evaluación de las causas de los eventos adversos o incidentes que afectan la seguridad del paciente, en el hospital Central de la Policia de Enero de 2012 a diciembre 2013. Universidad del Rosario; 2015.

40. McLeod M, Ahmed Z, Barber N, Dean Franklin B. A national survey of inpatient medication systems in English NHS hospitals. BMC Health Services Research. 2014;93(14):1-11. doi: 10.1186/1472-6963-14-93.

41. Orser BA, Hyland S, David U, Sheppard I, Wilson CR. Review article: Improving drug safety for patients undergoing anesthesia and surgery. Canadian Journal of Anesthesia. 2013;60(2):127-135. doi: 10.1007/s12630012-9853-y.

42. Pastó Cardona L, Masuet Aumatell C, Bara Oliván B, Castro Cels I, Clopés Estela A, Pàez Vives F, Schönenberger-Arnaiz JA, Gorgas Torner MQ, Codina Jané C. Estudio de incidencia de los errores de medicación en los procesos de utilización del medicamento: prescripción, transcripción, validación, preparación, dispensación y administración en el ámbito hospitalario. Farmacia Hospitalaria. 2009;33(5):257-268

43. Rowlands J, Yeager MP, Beach M, Patel HM, Huysman BC, Loftus RW. Video observation to map hand contact and bacterial transmission in operating rooms. American Journal of Infection Control. 2014;42(7):698701. doi: 10.1016/j.ajic.2014.02.021.

44. Gómez-Arnau JI, Otero MJ, Bartolomé A, Errando CL, Arnal D, Moreno AM, Puebla G., Marzal JM, Santa Úrsula JA, González R, Pérez M, García del Valle S, González A, Domínguez Gil A. Etiquetado de los medicamentos inyectables que se administran en anestesia. Revista Española de Anestesiología y Reanimación. 2011;58:375383. doi: 10.1016/S0034-9356(11)70087-1.

45. Gómez Arnau JI, Bartolomé A, García del Valle S, Puebla G, Santa Úrsula JA, Marzal JM, Arnal D, González A, González R. Errores de medicación y etiquetado de las jeringas. Revista Española de Anestesiología y Reanimación. 2013;60(Supl 1):27-33. doi: 10.1016/S00349356(13)70007-0.

46. Mellin-Olsen J, Staender S, Whitaker D, Smith A. The Helsinki Declaration on Patient Safety in Anaesthesiology. European Journal of Anaesthesiology. 2010;27(7):592597. doi: 10.1097/EJA.0b013e32833b1adf.

47. Merry AF, Shipp DH, Lowinger JS. The contribution of labelling to safe medication administration in anaesthetic practice. Best Practice \& Research Clinical Anaesthesiology. 2011;25(2):145-159. doi: 10.1097/ EJA.0b013e32833b1adf.

48. Escandón Vargas AZK. La importancia del control y prevención de enfermedades infecciosas en anestesiología. Revista Colombiana de Anestesiología. 2017;45(2): 69-77. doi: 10.1016/j.rca.2017.09.002.
49. Birkhead G, Hamilton TE, Kossover R, Perz J, Gangadharan D, Iskander J. CDC Grand rounds: preventing unsafe injection practices in the U.S. health-care system [Internet]. Atlanta; 2013. Disponible en: https://tinyurl. com/8v8dhdsj.

50. Ford K. Survey of syringe and needle safety among student registered nurse anesthetists: are we making any progress? American Association of Nurse Anesthetists. 2013;81(1):37-42.

51. King CA, Ogg M. Safe Injection Practices for Administration of Propofol. AORN Journal. 2012;95(3):365372. doi: 10.1016/j.aorn.2011.06.009.

52. Gounder P, Beers R, Bornschlegel K, Hinterland K, Balter S. Medication injection safety knowledge and practices among anesthesiologists: New York State, 2011. Journal of Clinical Anesthesia. 2013;25(7):521528. doi: 10.1016/j.jclinane.2013.04.013.

53. Zorrilla Vaca A, Arevalo JJ, Escandón Vargas K, Soltanifar D, Mirski MA. Infectious disease risk associated with contaminated propofol anesthesia, 1989-2014. Emerging infectious diseases. 2016;22(6):981-992. doi: 10.3201/eid2206.150376

54. Bennett SN, McNeil MM, Bland LA, Arduino MJ, ViIlarino ME, Perrotta DM, et al. Postoperative infections traced to contamination of an intravenous anesthetic, propofol. New England Journal of Medicine. 1995;333:147154. doi: 10.1056/NEJM199507203330303.

55. Muller AE, Huisman I, Roos PJ, Rietveld AP, Klein J, Harbers JBM, et al. Outbreak of severe sepsis due to contaminated propofol: lessons to learn. Journal of Hospital Infection. 2010;76(3):225-230. doi: 10.1016/j. jhin.2010.06.003.

56. Cole DC, Baslanti TO, Gravenstein NL, Gravenstein $\mathrm{N}$. Leaving more than your fingerprint on the intravenous line: a prospective study on propofol anesthesia and implications of stopcock contamination. Anesthesia and Analgesia. 2015;120(4):861-867. doi: 10.1213/ ANE.0b013e318292ed45.

57. Aspen Pharma Trading Limited. AEMPS Ficha Técnica Ultiva ${ }^{\circledR}$. Dublin; 2018.

58. United States Pharmacopeial. USP General Chapter $<795>$ Pharmaceutical Compounding: Nonsterile Preparations [Internet]. 2019 [citado 10 oct 2020]. Disponible en: www.usp.org.

59. Kishi D, Luiz R. Description of nosocomial infection prevention practices by anesthesiologists in a university hospital. Brazilian Journal of Anesthesiology. 2011;61(2):177187. doi: 10.1016/S0034-7094(11)70022-8.

60. Wolf ZR, Hughes RG. Best practices to decrease infusion-associated medication errors. Journal of Infusion Nursing. 2019;42(4):183-192. doi: 10.1097/ NAN.0000000000000329.

61. Schnock KO, Dykes PC, Albert J, Ariosto D, Call R, Cameron C, Carroll DL, Drucker AG, Fang L, Garcia Palm CA, Husch MM, Maddox RR, McDonald N, Mc- 
Guire J, Rafie S, Robertson E, Saine D, Sawyer MD, Smith LP, Stinger KD, Vanderveen TW, Wade E, Yoon CS, Lipsitz S, Bates DW. The frequency of intravenous medication administration errors related to smart infusion pumps: a multihospital observational study. British Medical Journal. 2017;26(2):131-140. doi: 10.1136/bmjqs2015-004465.

62. Timmerman AM, Oliveira Martens SM, Snijder RA, Nieman AK, Egberts TC. How to use current practice, risk analysis and standards to define hospital-wide policies on the safe use of infusion technology. Biomedical Engineering / Biomedizinische Technik. 2015;60(4):381387. doi: 10.1515/bmt-2014-0147.

63. Organización Mundial de la Salud, Safe Injection Global Network. Carpeta de material sobre seguridad de las inyecciones y los procedimientos conexos. Ginebra: OMS; 2010.

64. Hemingway CJ, Malhotra S, Almeida M, Azadian B, Yentis SM. The effect of alcohol swabs and filter straws on reducing contamination of glass ampoules used for neuroaxial injections. Anaesthesia. 2007;62:286-288. doi: 10.1111/j.1365-2044.2007.04977.x.

65. Woodbury A, Knight K, Fry L, Margolias G, Lynde GC. A survey of anesthesiologist and anesthetist attitudes toward single-use vials in an academic medical center. Journal of Clinical Anesthesia. 2014;26(2):125-130.

66. Ruiz Abellón J, Subiela García JA, Díaz Martínez AM. Compatibilidad entre fármacos por vía intravenosa. Revista Enfermería Docente. 2015;103:10-24.

67. Westbrook JI, Rob MI, Woods A, Parry D. Errors in the administration of intravenous medications in hospital and the role of correct procedures and nurse experience. British Medical Journal. 2011;20:1027-1034. doi: 10.1136/bmjqs-2011-000089.

68. Nikolova I, Tencheva J, Voinikov J, Petkova V, Danchev N, Nikolova I. Metamizole: a review profile of a well-known "forgotten" drug. Part I: Pharmaceutical and nonclinical profile pharmaceutical biotechnology. Biotechnology \& Biotechnological Equipment. 2012;26(6):3329-3337. doi: 10.5504/BBEQ.2012.0089.

69. Rabadán Anta MT, Flores Baeza M, Cayuela Fuentes J, Cevidades Lara M., Valvuena Moya R, Ruiz Morales MT, Rodríguez Mondéjar M, Cervantes Martínez MD. Interacciones medicamentosas en la administración de fármacos dentro del proceso de enfermería. Enfermería Global. 2002;1:1-23.

70. Regina Secoli S, Perez Esquirol E, Heras Matellan MJ Vendrell-Bosh L, Ballaríın-Alins E. Incompatibilidades en la terapia intravenosa: ¿qué hacer para prevenirlas? Enfermería Clínica. 2009;19(6):349-353.

71. Abellón Ruiz J, Subiela García JA, Díaz Martínez AM. Compatibilidad entre fármacos por vía intravenosa. Revista Enfermería Docente. 2015;(103):19-24.

72. Nascimento MA, Freitas K, Oliveira CGS. Erros na administração de medicamentos na prática assistencial da equipe de enfermagem: uma revisão sistemática. Ciências Biológicas e da Saúde. 2016;3(3):241-256.

73. Alliance for Patient Safety. Hand hygiene technical reference manual: to be used by health-care. Geneva: World Health Organization; 2009.

74. Mendes JR, Lopes MCBT, Vancini Campanharo CR, Okuno MFP, Batista REA. Types and frequency of errors in the preparation and administration of drugs. Einstein (São Paulo). 2018;16(3):1-6. doi: 10.1590/S1679$45082018 \mathrm{AO} 4146$.

75. American Association of Nurse Anesthetists. Safe injection guidelines for needle and syringe use [Internet]. 2014 [citado 10 oct 2020]. Disponible en: https://tinyurl. com/f7xwbsjr.

76. Ford K. Survey of syringe and needle safety amoung student registered nurse anesthetists: are we making any progress? American Association of Nurse Anesthetists. 2013;81(1):37-42.

77. The Joint Commission. 2019 National Patient Safety Goals [Internet]. 2019. Disponible en: https://tinyurl. com/w4xnvfw.

78. Sistema Español de Notificación en Seguridad en Anestesia y Reanimación, Instituto para el Uso Seguro de los Medicamentos. Recomendaciones para el etiquetado de los medicamentos inyectables que se administran en Anestesia [Internet]. 2011 [citado 10 oct 2020]. Disponible en: https://tinyurl.com/54uyk6jt.

79. Ramírez Madrigal MC, Anduquia Vásquez PA, Alvarez Cano LM, Forero Pulido C, Beltrán Salazar OA, Cardozo García Y, et al. El cuidado de enfermería en la administración segura de medicamentos. Bogotá, D.C.; 2012.

80. Dhawan I, Tewari A, Sehgal S, Chandra A. Medication errors in anesthesia: unacceptable or unavoidable? Brazilian Journal of Anesthesiology. 2017;67(2):184192. doi: 10.1016/j.bjane.2015.09.006.

81. Karthikeyan M, Lalitha D. A prospective observational study of medication errors in the general medicine department in a tertiary care hospital. Drug Metabolism Drug Interactions. 2013;28(1):13-21. doi: 10.1515/ dmdi-2012-0032.

82. Machado Alba JE, Moreno Gutiérrez PA, Moncada Escobar CJ. Hospital medication errors in a pharmacovigilance system in Colombia. Farmacia Hospitalaria. 2015;39(6):338-349. doi: 10.7399/fh.2015.39.6.8899.

83. Jornet Montaña S, Canadell Vilarasa L, Calabuig Muñoz M, Riera Sendra G, Vuelta Arce M, Bardají Ruiz A, Gallart Mora MJ. Detección y clasificación de errores de medicación en el Hospital Universitario Joan XXIII. Farmacia Hospitalaria. 2004;28(2):90-96.

84. Grigg EB, Martin LD, Ross FJ, Roesler A, Rampersad SE, Haberkern C, Low DKW, Carlin K, Martin LD. Assessing the impact of the anesthesia medication template on medication errors during anesthesia: a prospective study. Anesthesia and Analgesia. 2017;124(5):16171625. doi: 10.1213/ANE.0000000000001823. 
85. Cooper L, Nossaman B. Medication errors in anesthesia: a review. International Anesthesiology Clinics. 2013;51(1):1-12. doi: 10.1097/AIA.0b013e31827d6486.

86. Santell JP, Hicks RW, McMeekin J, Cousins DD. Medication errors: experience of the United States Pharmacopeia (USP) MEDMARX reporting system. Journal of Clinical Pharmacology. 2003;43(7):760-767.
87. Cousins DD, Heath WM. The national coordinating council for medication error reporting and prevention: promoting patient safety and quality through innovation and leadership. The Joint Commission Journal on Quality and Patient Safety. 2008;34(12):700-2. doi: 10.1016/ s1553-7250(08)34091-4.

\section{FORMA DE CITAR}

Villanueva AH, López Gutiérrez JJ. Análisis de errores con medicamentos en pacientes ingresados en salas de cirugía y recuperación posanestésica de un hospital de alta complejidad en Bogotá, Colombia. Salud Colectiva. 2021;17:e3155. doi: 10.18294/sc.2021.3155.

Recibido: 8 sep 2020 | Versión final: 8 feb 2021 | Aprobado: 1 mar 2021 | Publicado en línea: 26 abr 2021

Esta obra está bajo una licencia de Creative Commons Reconocimiento-NoComercial 4.0 Internacional. Reconocimiento - Permite copiar, distribuir y comunicar públicamente la obra. A cambio, se debe reconocer y citar al autor original. No Comercial — Esta obra no puede ser utilizada con finalidades comerciales, a menos que se obtenga el permiso. 